\title{
Can Parkinson's disease be cured by stimulating neurogenesis?
}

\author{
Thomas Foltynie \\ Sobell Department of Motor Neuroscience, UCL Institute of Neurology, National Hospital for Neurology and Neurosurgery, London, United Kingdom.
}

\begin{abstract}
There have been many attempts at slowing down or even reversing the neurodegenerative process of Parkinson's disease (PD). To date, there are no treatments of proven value in this regard. One underexplored route to slow the neurodegenerative process is the use of agents that may stimulate neurogenesis in the subventricular zone. In animal models of PD, PDGF-BB has been shown to restore/protect against dopaminergic deficits caused by neurotoxins via increased neurogenesis in the subventricular zone. Previous work suggests that these new cells are not themselves dopaminergic but have trophic effects on residual dopaminergic cells in the substantia nigra. In this issue of the $J C I$, Paul et al. evaluate this agent in individuals with PD and show that i.c.v. administration of PDCF-BB is safe and well tolerated. This study lays the foundation for formal dose-finding studies and clinical trials to assess the efficacy of this agent as a potential neuroprotective treatment for PD.
\end{abstract}

\section{Growth factors for prevention of neurodegeneration}

The use of growth factors to try to prevent neurodegeneration is not a new concept. Administration of glial cell-derived neurotrophic factor (GDNF) has long since been shown to increase the number of tyrosine hydroxylase-positive (TH-positive) cells in the striatum, an effect accompanied by positive behavioral effects in a range of animal models of Parkinson's disease (PD). Unfortunately, GDNF, which has had encouraging preclinical effects in these models, has had mixed results in the clinic. Infusion of high doses of GDNF into the cerebral ventricles of 50 patients for up to 20 months failed to show any advantage in Unified PD Rating Scale scores when compared with placebo. Initially, this result was interpreted as a failure of GDNF to penetrate into the striatum when administered via the i.c.v. route (1). Subsequently, beneficial effects were observed when GDNF was delivered by continuous infusion directly into the posterior putamen in 2 "open-label" trials $(2,3)$; however, these same results could not be replicated in a double-blind trial (4). Further concerns were raised regarding the detection of anti-GDNF antibodies in serum following treatment and the possibility that GDNF may be associated with cerebellar toxicity (5). While there is ongoing interest in GDNF as a potential neuroprotective treatment (6), further concerns regarding the potential efficacy of GDNF in human PD have emerged recently in response to the observation that the GDNF "ret" receptor is downregulated in $\mathrm{PD}$, which may minimize the possibility of a cellular response to this specific growth factor (7).

In recent years, interest has grown in the study of PDGF-BB and its effects on neurogenesis in the subventricular zone (SVZ), which is a primary site of adult neurogenesis. PDGF-BB is a growth factor of the PDGF/VEGF family that exists in var-

Related Article: p. 1339

Conflict of interest: The author has declared that no conflict of interest exists.

Reference information: J Clin Invest. 2015;125(3):978-980. doi:10.1172/JCI80822.

ious isoforms, each with different abilities to bind to tyrosine kinase PDGF receptors. PDGF-BB is a dimer of the B isoform, which is upregulated following dopaminergic cell injury presumably as a compensatory physiological response (8). PDGF receptor-expressing neuronal precursors persist in the SVZ into adulthood $(9,10)$, and infusions of PDGF-BB into the ventricles of adult rats with dopaminergic lesions is reproducibly associated with an increase in newly formed cells in the striatum and substantia nigra (SN) and functional recovery $(9,11)$. These effects can be blocked by the mitosis inhibitor Ara-C (9), strongly indicating that the behavioral and histological effects seen relate, at least in part, to an action of PDGF-BB on the genesis of new cells. It is of important mechanistic interest that, while these newly generated cells include NeuN-positive neurons, they do not express $\mathrm{TH}$, which is a prerequisite enzyme for dopamine synthesis; therefore, the effects of PDGF-BB-induced neurons are seemingly not due to the assumption of a dopaminergic phenotype.

\section{Tolerability of PDGF-BB in small PD cohort}

In this issue, Paul et al. have further advanced clinicalstudyofneurotrophicfactors in PD by exploring whether PDGF-BB may have potential as an alternative neuroprotective/neurorestorative agent (12). Twelve patients with PD of moderate severity were assessed for tolerability of 3 different doses of PDGF-BB infused into the cerebral ventricles for a 2 -week period compared with tolerability of infused placebo. As this was a "first-in-human" study, Paul and colleagues used a gradual dose-escalating design and a 3:1 ratio of patients receiving active treatment to those receiving placebo.

Adverse events were recorded carefully and, overall, provide support for the claim made by Paul and colleagues that this approach is well tolerated by patients with PD. Minor problems were associated 
with the surgical device, but none of these interfered with successful completion of the study. Furthermore, no anti-PDGF antibodies were detected in the sera of PDGF-BB-treated individuals during the follow-up period. The exposure time to PDGF infusion was brief and future studies will need to carefully evaluate whether longer exposure periods are accompanied by similar safety and tolerability.

The study by Paul and colleagues was not designed or powered to measure efficacy; therefore, it is far too early to reach any conclusions as to whether this agent may ultimately play a role in the treatment of patients with PD. Unified PD Rating Scale scores were no different between the active treatment and placebo groups; a result that should be interpreted as simply providing further reassurance regarding the safety of the PDGF-BB administration. From an optimistic viewpoint, however, Paul et al. did observe a notable effect on dopamine transporter binding (an estimate of the number of available dopaminergic presynaptic terminals) when the patient data were analyzed with an adjustment for baseline severity and the interval between scans.

\section{Remaining questions and future directions}

There remains some degree of uncertainty regarding the extent to which effects of GDNF and PDGF-BB in animal models are overlapping or distinct. It should be noted that infusion of GDNF appears to lead to glial cell proliferation in the $\mathrm{SN}$, without evidence of neurogenesis, and that the beneficial effects of GDNF observed in the laboratory appear to be mediated through upregulation of $\mathrm{TH}$ in existing neurons rather than neurogenesis (13). With respect to PDGF-BB, the compelling effects of this treatment on SVZ neurogenesis have been seen in parallel with relevant behavioral improvements, and the study by Paul et al. now provides additional reassurance that PDGF-BB can be safely administered to patients with PD. It remains to be seen, however, whether the mechanism of action of PDGF-BB is purely via SVZ neurogenesis. Another recent study showed that pretreatment of a glioblastoma line with this agent protects against rotenone (a potent complex 1 inhibitor) toxicity through a reduction in oxidative stress and preservation of mitochondrial function (14). Furthermore, PDGF is known to have a role in the stimulation of survival signals such as AKT (15), a feature that has potential to be highly relevant to PD pathogenesis. Alongside recent concerns that the GDNF ret receptor is downregulated in human PD (7), it will be important for future studies to determine whether PDGF receptor expression and/or responsiveness is altered in the presence of excessive and/ or mutated $\alpha$-synuclein.

Some might say that the use of growth factor infusions for PD is "too little, too late." However, postmortem evaluations of patients at varying stages of PD suggest that there are residual pigmented cells in the $\mathrm{SN}$ for up to 10 years after diagnosis $(16,17)$, while the number of TH-positive cells that remain in the striatum may be substantially lower (18). This observation does not necessarily indicate cell death, as these cells may remain salvageable, with the potential to exert a functional role on neurotransmission following appropriate growth factor support.

The quest for identification of a neuroprotective agent for $\mathrm{PD}$ requires further investigations of multiple agents to be conducted in parallel (19). Moreover, in vitro and in vivo laboratory work needs to continue to reassess encouraging avenues of interest in the most relevant animal models of PD that can help provide information on which agents to pursue in patient populations, based on greater insight into their true mechanisms of action (20). Alongside the need for ongoing laboratory work, there is an urgent need to accelerate the identification of efficacious neuroprotective drugs in $\mathrm{PD}$, which necessitates the conduct of small scale clinical trials, which can inform on safety, tolerability, optimal dosing strategies, and required duration of exposure for formal efficacy trials.

\section{Acknowledgments}

The UCL Institute of Neurology is partly funded by the Department of Health National Institute for Health Research Biomedical Research Centres funding scheme. T. Foltynie has support from the Parkinson's Appeal and holds grants from the Michael J. Fox Foundation (TPP 2013), the European Union FP-7 scheme (TRANSEURO), and the Brain Research Trust (PAR11141).
Address correspondence to: Thomas Foltynie, Sobell Department of Motor Neuroscience, UCL Institute of Neurology, Box 146, National Hospital for Neurology and Neurosurgery, Queen Square, London, WC1N 3BG, United Kingdom. Phone: 44.203.448.8726; E-mail: T.Foltynie@ucl.ac.uk.

1. Nutt JG, et al. Randomized, double-blind trial of glial cell line-derived neurotrophic factor (GDNF) in PD. Neurology. 2003;60(1):69-73.

2. Gill SS, et al. Direct brain infusion of glial cell line-derived neurotrophic factor in Parkinson disease. Nat Med. 2003;9(5):589-595.

3. Slevin JT, Gerhardt GA, Smith CD, Gash DM, Kryscio R, Young B. Improvement of bilateral motor functions in patients with Parkinson disease through the unilateral intraputaminal infusion of glial cell line-derived neurotrophic factor. J Neurosurg. 2005;102(2):216-222.

4. Lang AE, et al. Randomized controlled trial of intraputamenal glial cell line-derived neurotrophic factor infusion in Parkinson disease. Ann Neurol. 2006;59(3):459-466.

5. Hovland DN Jr, et al. Six-month continuous intraputamenal infusion toxicity study of recombinant methionyl human glial cell line-derived neurotrophic factor (r-metHuGDNF) in rhesus monkeys. Toxicol Pathol. 2007;35(5):676-692.

6. Patel NK, Pavese N, Javed S, Hotton GR, Brooks DJ, Gill SS. Benefits of putaminal GDNF infusion in Parkinson disease are maintained after GDNF cessation. Neurology. 2013;81(13):1176-1178.

7. Decressac M, Kadkhodaei B, Mattsson B, Laguna A, Perlmann T, Björklund A. $\alpha$-Synuclein-induced down-regulation of Nurr1 disrupts GDNF signaling in nigral dopamine neurons. Sci Transl Med. 2012;4(163):163ra156.

8. Funa K, et al. Enhanced synthesis of platelet-derived growth factor following injury induced by 6-hydroxydopamine in rat brain. Neuroscience. 1996;74(3):825-833.

9. Zachrisson $\mathrm{O}$, et al. Restorative effects of platelet derived growth factor-BB in rodent models of Parkinson's disease. J Parkinsons Dis. 2011;1(1):49-63.

10. Moore L, Bain JM, Loh JM, Levison SW. PDGFresponsive progenitors persist in the subventricular zone across the lifespan. ASN Neuro. 2014;6(2):65-81.

11. Mohapel P, Frielingsdorf H, Häggblad J, Zachrisson O, Brundin P. Platelet-derived growth factor (PDGF-BB) and brain-derived neurotrophic factor (BDNF) induce striatal neurogenesis in adult rats with 6-hydroxydopamine lesions. Neuroscience. 2005;132(3):767-776

12. Paul G, et al. Safety and tolerability of intracerebroventricular PDGF-BB in Parkinson's disease patients. JClin Invest. 2015;125(3):1339-1346.

13. Chen Y, Ai Y, Slevin JR, Maley BE, Gash DM. Progenitor proliferation in the adult hippocampus and substantia nigra induced by glial cell line-derived neurotrophic factor. Exp Neurol. 
2005;196(1):87-95.

14. Cabezas R, Avila MF, González J, El-Bachá RS, Barreto GE. PDGF-BB protects mitochondria from Rotenone in T98G cells [published online ahead of print December 17, 2014]. Neurotox Res. doi:10.1007/s12640-014-9509-5.

15. Funa K, Sasahara M. The roles of PDGF in development and during neurogenesis in the normal and diseased nervous system. J Neuroimmune Pharmacol. 2014;9(2):168-181.
16. Fearnley JM, Lees AJ. Ageing and Parkinson's disease: substantia nigra regional selectivity. Brain. 1991;114(pt 5):2283-2301.

17. Pakkenberg B, Møller A, Gundersen HJ, Mouritzen Dam A, Pakkenberg H. The absolute number of nerve cells in substantia nigra in normal subjects and in patients with Parkinson's disease estimated with an unbiased stereological method. J Neurol Neurosurg Psychiatry. 1991;54(1):30-33.
18. Kordower JH, et al. Disease duration and the integrity of the nigrostriatal system in Parkinson's disease. Brain. 2013;136(pt 8):2419-2431.

19. Athauda D, Foltynie T. The ongoing pursuit of neuroprotective therpaies in Parkinson disease. Nat Rev Neurol. 2015;11(1):25-40.

20. Luk KC, et al. Pathological $\alpha$-synuclein transmission initiates Parkinson-like neurodegeneration in nontransgenic mice. Science. 2012;338(6109):949-953. 